\title{
OPTIMAL INFINITE HORIZON CONTROL UNDER A LOW DATA RATE ${ }^{2}$
}

\author{
Girish N. Nair $^{1}$ Minyi Huang Robin J. Evans \\ Department of Electrical and Electronic Engineering \\ University of Melbourne, VIC 3010, Australia
}

\begin{abstract}
This paper considers the optimal control of linear systems where measurement data is transmitted from the plant output to the controller over a noiseless communication channel with limited instantaneous data rate. The cost is defined to be the average, over a random initial state, of the usual infinite horizon quadratic regulation criterion, and the number of bits transported by the channel during each sampling interval is bounded. Several fundamental properties of the optimal cost functional are derived for initial state densities that satisfy a mild moment condition. Using these properties, precise expressions for the optimal cost and policy are obtained assuming a uniformly distributed initial state. These expressions agree with the classical optimal LQR results in the high data rate limit and with recent minimum rate results in the low rate regime. Extensions to the case of non-uniform densities and vector-valued states are discussed.
\end{abstract}

Keywords: Optimal Control, Communication Channels, Quantization

\section{INTRODUCTION}

In recent years, there has been an immense amount of research into the interaction between communication and feedback control objectives in various settings (see, e.g. (Special Issue on Networked Control Systems, 2004)). This activity has primarily been motivated by applications in which multiple sensors and actuators communicate over non-ideal communication channels that may be shared, have low data rates, suffer from timevarying or random delays, introduce errors and so on. These limitations can have a significant adverse effect on the control objectives and need to be explicitly addressed when analysing and designing such systems.

In order to be able to analyse a full networked control system, it is necessary to have a thorough

\footnotetext{
1 Email: gnair@ee.unimelb.edu.au

2 This work was partially supported by Australian Research Council grant DP0345044 and NICTA Ltd.
}

understanding of the effects that these communication constraints have in the simplest possible situation where one sensor communicates to one controller over a single channel. Continuing a line of inquiry begun in (Delchamps, 1990; Wong and Brockett, 1999; Brockett and Liberzon, 2000; Baillieul, 2001), this paper focuses on communication channels in which the critical limitation is low data rate, in bits/sample. From a practical point of view, this emphasis is valid in systems where the sampling interval is sufficiently long that communication delays have negligible effect. In addition, many control network protocols are implemented with a standard overhead of error correction coding which significantly reduces the number of bit errors. It is well-known now that at low data rates, control performance degrades drastically and even the most basic objective such as stability can become impossible. Much of the theoretical research in this area has focused on determining minimum feedback data rates for various notions of stabilisability (Wong 
and Brockett, 1999; Baillieul, 2001; Nair and Evans, 2000; Tatikonda and Mitter, 2000; Nair and Evans, 2003; Nair and Evans, 2004; Li and Baillieul, 2004), and precise expressions have been derived in terms of the unstable open-loop eigenvalues of the plant.

However, a similarly precise characterisation of the effect of a finite data rate on achievable performance is generally lacking. A universal lower bound on the average state power of stochastic linear systems was derived in (Nair and Evans, 2004), and in (Tatikonda et al., 2004) a separation principle was derived for Gaussian linear plants when the control is a linear function of a state estimate. With regard to deterministic plants, upper bounds on performance for specific coding and control policies have been obtained, under different performance criteria (Petersen and Savkin, 2001; Ishii and Francis, 2003; Liberzon, 2003) and, in (Fagnani and Zampieri, 2003; Fagnani and Zampieri, 2004a), the relationships between mean entrance times, invariant set contraction ratios and the number of quantisation levels were characterised for memorlyess quantisers.

This paper studies the optimal data-rate-limited control of noiseless linear systems where the coder and controller are permitted to be time-varying with memory. The performance cost adopted is the average, over a random initial state, of the usual quadratic regulation criterion. In sec. 3, the infimum cost is treated as a functional of the initial state probability density and several of its fundamental properties are obtained under a mild moment condition. Using these properties, precise formulae for the optimal cost and policy are derived in sec. 4 when the initial state is uniformly distributed. These expressions agree with the classical optimal LQR results in the high data rate limit and with the minimum rate results mentioned above. Although lower and upper bounds on infinite-horizon LQR performance under communication constraints are known (Fagnani and Zampieri, 2004b), to the best of the authors' knowledge these results represent the first exact characterisation of optimal data-rate-limited performance in any setting. Extensions to nonuniform distributions are also discussed.

\section{FORMULATION}

The notation used in this paper is first defined. Sequences $\left\{s_{j}\right\}_{j=0}^{n}$ (of real numbers, discrete symbols, or functions) are denoted $\tilde{s}_{n}$, with $\tilde{s}_{-1}$ being the empty sequence and $\tilde{s}:=\left\{s_{j}\right\}_{j=0}^{\infty}$. All random variables (rv's) are assumed to exist in a common probability space and are written in uppercase, with their realisations represented by the corresponding lower-case letters. The probability density function (pdf) of a random variable $X$ is written as $\mathrm{f}_{X}(\cdot)$ and its expected value as $\mathrm{E}\{X\}$. Finally, the conditional pdf and expectation of $X$ given an event $S=s$ are written as $\mathrm{f}_{X \mid s}(\cdot)$ and $\mathrm{E}_{s}\{X\}$ respectively.

For given $a, b \in \mathbb{R}$, consider a scalar, discrete-time, linear input-output system

$$
x_{t+1}=a x_{t}+b u_{t} \in \mathbb{R}, \quad t \in[0,1,2, \ldots),
$$

which evolves from some initial condition $x_{0} \in \mathbb{R}$ with real-valued input sequence $\tilde{u}=\left\{u_{t}\right\}_{t=0}^{\infty}$. Assume that $x_{0}$ is a realisation of a random variable $X_{0}$, with known $\operatorname{pdf}_{X_{0}}$ and finite absolute $(2+\varepsilon)$ th moment $\mathrm{E}\left\{\left|X_{0}\right|^{2+\varepsilon}\right\}<\infty$ for some $\varepsilon>0$.

The definitions of the coder, channel and controller below are nearly identical to (Nair and Evans, 2003). Suppose that the sensor which observes the states of the system is connected to the controller via a digital channel onto which one symbol $s_{t}$ from a fixed, finite alphabet $\mathcal{S}$ is transmitted during the $(t+1)$ th sampling interval. Assuming that error coding or retransmission protocols are employed and that the propagation delay is not larger than one sampling interval, it is reasonable to suppose that each transmitted symbol is received without at the other end of the channel after a nominal delay of one sampling interval. The data rate of the channel may be defined as $R:=\log _{2}|\mathcal{S}|$. In order to focus on the performance degradation caused by the finite data rate, it is assuming that there are no computational constraints at the coder or controller. Each symbol $s_{t}$ transmitted by the coder may then depend in a time-varying way on all past and present states and past symbols,

$$
s_{t}=\gamma_{t}\left(\tilde{s}_{t-1}, \tilde{x}_{t}\right) \in \mathcal{S}, \quad \forall t \in[0,1,2, \ldots), .
$$

where $\gamma_{t}$ is measurable. At time $t$ the controller has the symbol sequence $\tilde{s}_{t-1}$ available and can then generate a control signal of the general form

$$
u_{t}=\delta_{t}\left(\tilde{s}_{t-1}\right) \quad \forall t \in[0,1,2, \ldots),
$$

via another measurable, time-varying function $\delta_{t}$. As current analog-digital and digital-analog converters often have adjustable bias levels and dynamic ranges, the assumption of time-varying, recursive coders and controllers is not an impractical one.

Define the coder-controller as $(\tilde{\gamma}, \tilde{\delta})$, the ordered pair of coder and controller mapping sequences, and denote the set of all possible coder-controllers for the given channel alphabet $\mathcal{S}$ by $\mathcal{C}$. An important assumption made throughout this paper is that both coder and controller know the initial pdf of $X_{0}$ exactly, though of course the controller cannot precisely know the specific realisation $x_{0}$.

The performance cost considered in this paper is the average of the classical quadratic regulation criterion, 


$$
J\left(\mathrm{f}_{X_{0}}, \tilde{\gamma}, \tilde{\delta}\right):=\sum_{t=0}^{\infty} \mathrm{E}\left\{X_{t+1}^{2}+r U_{t}^{2}\right\}
$$

where $r \geq 0$ is a selected constant. Note that an average over the initial state pdf is taken because the controller here does not know the exact initial state, whereas the unaveraged LQR-optimal policy would not necessarily be independent of $x_{0}$ as in the classical case, due to the nonlinearity induced by the digital channel. It can be shown using (Nair and Evans, 2003) that, unlike the deterministic classical LQR problem, a finite cost here is possible iff $|\mathcal{S}|>\max \{|a|, 1\}$, provided also that $X_{0}$ has a finite absolute moment $\mathrm{E}\left\{\left|X_{0}\right|^{2+\varepsilon}\right\}<\infty$ for some $\varepsilon>2$. Assuming throughout this paper that these conditions hold, the infimum cost

$$
J_{*}\left(\mathrm{f}_{X_{0}}\right):=\inf _{(\tilde{\gamma}, \tilde{\delta}) \in \mathcal{C}} J\left(\mathrm{f}_{X_{0}}, \tilde{\gamma}, \tilde{\delta}\right)
$$

is then well-defined.

The objectives of this paper are twofold. In the following section, certain fundamental properties of the functional $J_{*}$ are presented, in particular the effects of scaling and shifting the initial state distribution, and a recursive expression for $J_{*}$ is obtained. Using these results, it is then shown in sec. 4 that explicit formulae can be derived for $J_{*}\left(f_{X_{0}}\right)$ and the associated optimal codercontroller, for the case of uniformly distributed $X_{0}$.

\section{GENERAL PROPERTIES OF $J_{*}$ FUNCTIONAL}

In this section several fundamental characteristics of the infimum cost functional $J_{*}$ are presented. In order to ensure a finite infimum cost it is assumed that $\mathrm{E}\left\{\left|X_{0}\right|^{2+\varepsilon}<\infty\right.$ for some $\varepsilon>0$ and that the alphabet size $|\mathcal{S}|>\max \{|a|, 1\}$. Due to a lack of space, proofs of these properties are omitted.

The first property describes the effect of stretching or compressing the distribution by a constant factor. A similar scaling law trivially applies to the unaveraged LQR cost of any linear controller, but in the nonlinear channel-constrained case it can only be generally proven for the optimal average cost functional.

Property 1. (Scaling). For any real constant $c \neq 0$ and any random variable $X \in \mathbb{R}$ with finite moment $\mathrm{E}\left\{|X|^{2+\varepsilon}\right\}<\infty$ for some $\varepsilon>0$,

$$
J_{*}\left(\mathrm{f}_{c X}\right)=c^{2} J_{*}\left(\mathrm{f}_{X}\right) .
$$

$\nabla$

The second property describes the effect on $J_{*}\left(\mathrm{f}_{X}\right)$ of shifting the distribution of $X$ by its mean.
Property 2. (Removing the Mean). For any random variable $X \in \mathbb{R}$ such that $\mathrm{E}\left\{|X|^{2+\varepsilon}\right\}<\infty$ for some $\varepsilon>0$,

$$
J_{*}\left(\mathrm{f}_{X}\right)=J_{*}\left(\mathrm{f}_{X-\bar{x}}\right)+p(\bar{x})^{2},
$$

where $\bar{x}:=\mathrm{E}\{X\}$ and $p \in \mathbb{R}_{+}$is the solution to the steady-state scalar Riccati equation

$$
p=\frac{a^{2}(p+1) r}{b^{2}(p+1)+r} .
$$

$\nabla$

The next result states that the class of codercontrollers can be constrained without extra cost to those that encode only the current state given past symbols, using coding regions in the form of real intervals. This structural result is vital in deriving the main result of the following section. It can be generalised to the general vector case, with the coding regions then being convex polyhedra rather than intervals.

Lemma 1. (Interval Coding). Let $X$ be absolutely continuous w.r.t. Lebesgue measure and have finite moment $\mathrm{E}\left\{|X|^{2+\varepsilon}\right\}<\infty$ for some $\varepsilon>0$. Then

$$
\begin{aligned}
J_{*}\left(\mathrm{f}_{X}\right) \equiv & \inf _{(\tilde{\gamma}, \tilde{\delta}) \in \mathcal{C}} J\left(\mathrm{f}_{X}, \tilde{\gamma}, \tilde{\delta}\right)=\inf _{(\tilde{\gamma}, \tilde{\delta}) \in \mathcal{C}^{\mathrm{i}}} J\left(\mathrm{f}_{X}, \tilde{\gamma}, \tilde{\delta}\right), \\
\text { where } \mathcal{C}^{\mathrm{i}}:= & \left\{(\tilde{\gamma}, \tilde{\delta}) \in \mathcal{C}: \gamma_{t}\left(\cdot, \tilde{x}_{t}\right) \equiv \eta_{t}\left(\cdot, x_{t}\right)\right. \text { s.t. } \\
& \left\{x \in \mathbb{R}: \eta_{t}\left(\tilde{c}_{t-1}, x\right)=c_{t}\right\} \text { is an } \\
& \text { interval } \left., \forall \tilde{c}_{t} \in \mathcal{S}^{t+1}\right\}
\end{aligned}
$$

$\nabla$

The theorem below exploits the properties above and standard dynamic programming arguments to yield a general recursion for the infimum cost functional $J_{*}$. The key point is that expression is independent of the controller and involves an infimum over only the coding function.

Theorem 1. Let the initial state $X_{0} \in \mathbb{R}$ of (1) have finite moment $\mathrm{E}\left\{\left|X_{0}\right|^{2+\varepsilon}\right\}<\infty$ for some $\varepsilon>0$. Then the infimum cost (5) satisfies

$$
\begin{aligned}
& J_{*}\left(\mathrm{f}_{X_{0}}\right)=a^{2}\left[\operatorname{var}\left\{X_{0}\right\}+p\left(\bar{x}_{0}\right)^{2}\right] \\
& +a^{2} \inf _{\gamma_{0}}\left\{\sum_{s_{0} \in \mathcal{S}} \mathrm{P}\left\{S_{0}=s_{0}\right\} J_{*}\left(\mathrm{f}_{X_{0}-\bar{x}_{0} \mid s_{0}}\right)\right\},
\end{aligned}
$$

where $p \in \mathbb{R}_{+}$is given by the Riccati equation (6), $\bar{x}_{0}:=\mathrm{E}\left\{X_{0}\right\}$ and the inf is over all measurable coding functions $\gamma_{0}: \mathbb{R} \rightarrow \mathcal{S}$ that take $x_{0} \mapsto s_{0}$. $\nabla$

As a by-product of the proof of the preceding theorem, the following result can also be shown: 
Theorem 2. (Certainty Equivalence). For any fixed coder $\tilde{\gamma}$, the unique optimal control law for (1) is given by

$$
u_{t}^{*}=\delta_{t}^{*}\left(\tilde{s}_{t-1}\right):=-\frac{b p}{a r} \mathrm{E}_{\tilde{s}_{t-1}}\left\{X_{t}\right\} .
$$

If at time $t \in[0,1,2, \ldots)$ there exists a globally optimal coding function $\gamma_{t}^{*}: \mathcal{S}^{t} \times \mathbb{R}^{t+1} \rightarrow \mathcal{S}$ taking $\left(\tilde{s}_{t-1}, \tilde{x}_{t}\right) \mapsto s_{t}$, then it must satisfy the (controller-independent) minimisation

$$
\begin{aligned}
& \gamma_{t}^{*} \in \operatorname{Arg} \min _{\gamma_{t}} \\
& \left\{\sum _ { s _ { t } \in \mathcal { S } } \mathrm { P } \{ S _ { t } = s _ { t } | \tilde { s } _ { t - 1 } \} J _ { * } \left(\mathrm{f}_{\left.\left.\left(X_{t}-\mathrm{E}_{\tilde{s}_{t-1}}\left\{X_{t}\right\}\right) \mid \tilde{s}_{t}\right)\right\}}\right.\right.
\end{aligned}
$$

$\nabla$

A finite-horizon analogue of this result was given in (Yuksel et al., 2004). As $u_{t}=-b p /(a r) x_{t}$ is the optimal control policy when no communication constraint is present, (8) confirms that the certainty-equivalence principle applies in the infinite-horizon problem. The inclusion (9) implies that optimal coding laws are determined only by the conditional distribution of the current state given past symbols, and thus can be restricted to encode only the current state given past symbols. Note that this inclusion and the main theorem are properties of the global infimum cost and do not assume a fixed controller.

Despite its recursive nature, using Theorem 1 to obtain explicit expressions for the infimum cost is difficult, because the form of the functional $J_{*}$ for a given initial state distribution will generally not remain the same after being conditioned on an interval. If tractable approximations or upperbounds are available for $J_{*}\left(\mathrm{f}_{\left(X_{t} \mid \tilde{s}_{t}\right.}\right),(8)$ - (9) provide a method to systematically design suboptimal coder-controllers with guaranteed cost. However, in those special cases where $J_{*}$ does retain the same form after conditioning, Theorem 1 can be exploited to derive exact formulae for the optimal cost and policy. This is illustrated in the following section.

\section{EXPLICIT SOLUTION FOR UNIFORM $X_{0}$}

In this section, it is shown that explicit formulae for the optimal cost $J_{*}\left(\mathrm{f}_{X_{0}}\right)$ and coder-controller can be derived when $X_{0}$ is distributed uniformly on a given interval. In deriving these expressions, the general properties obtained in the previous section are exploited, together with the following simple observation.

Lemma 2. Let $X$ be uniformly distributed with mean zero on a given interval. Then

$$
J_{*}\left(\mathrm{f}_{X}\right) \equiv \alpha \operatorname{var}\{X\},
$$

where $\alpha \in \mathbb{R}_{+}$is a constant that depends only on the parameters $a, b$ of the system (1), the coding alphabet size $|\mathcal{S}|$ and the control weight $r$ of (4).

\section{Proof: Omitted.}

Solving the optimal channel-constrained average LQR problem for a uniform distribution is therefore equivalent to determining the parameter $\alpha$. When combined with Theorem 1 and Properties 1 and 1 , this observation leads to the result below.

Theorem 3. Let the initial state $X_{0}$ of (1) be uniformly distributed on some given interval. The minimum cost (5) is then given by

$$
\begin{aligned}
& J_{*}\left(\mathrm{f}_{X_{0}}\right)=p\left(\bar{x}_{0}\right)^{2}+ \\
& a^{2}\left[1+p+\frac{a^{2}(1+p)-p}{|\mathcal{S}|^{2}-a^{2}}\right] \operatorname{var}\left\{X_{0}\right\},
\end{aligned}
$$

where $\bar{x}_{0}:=\mathrm{E}\left\{X_{0}\right\},|\mathcal{S}|$ is the size of the coding alphabet $\mathcal{S}$, and $p$ is the solution to the Riccati equation (6) .

Proof: By Lemma 1, the function $\gamma_{0}$ can be constrained without additional cost to have coding regions in the form of intervals, so $\left\{x_{0} \in \mathbb{R}: s_{0}=\right.$ $\left.\gamma_{0}\left(x_{0}\right)=s\right\}$ can be assumed to be an interval, $\forall s \in \mathcal{S}$. Note that since the support of $\mathrm{f}_{X_{0}}$ is an interval, that of $\mathrm{f}_{X_{0} \mid s_{0}}$ must also be an interval, and also that since $f_{X_{0}}$ is constant on its support so is $f_{X_{0} \mid s_{0}}$. Hence, the conditional pdf $f_{X_{0} \mid s_{0}}$ is necessarily uniform on some interval, with mean $\mu\left(s_{0}\right):=\mathrm{E}_{s_{0}}\left\{X_{0}\right\}$ and variance

$$
\operatorname{var}_{s_{0}}\left\{X_{0}\right\}:=\mathrm{E}_{s_{0}}\left\{\left[X_{0}-\mu\left(s_{0}\right)\right]^{2}\right\} \equiv \mathrm{E}_{s_{0}}\left\{X_{0}^{2}\right\}-\mu\left(s_{0}\right)^{2} \text {. }
$$

From Property 2 and Lemma 2,

$$
\begin{aligned}
J_{*}\left(\mathrm{f}_{X_{0}-\bar{x}_{0} \mid s_{0}}\right)= & J_{*}\left(\mathrm{f}_{X_{0}-\mu\left(s_{0}\right) \mid s_{0}}\right)+p\left[\mu\left(s_{0}\right)-\bar{x}_{0}\right]^{2}, \\
\equiv & \alpha \operatorname{var}_{s_{0}}\left\{X_{0}\right\}+p \mu\left(s_{0}\right)^{2} \\
& +p\left[\left(\bar{x}_{0}\right)^{2}-2 \bar{x}_{0} \mu\left(s_{0}\right)\right], \\
= & (\alpha-p) \operatorname{var}_{s_{0}}\left\{X_{0}\right\}+p \mathrm{E}_{s_{0}}\left\{X_{0}^{2}\right\} \\
& +p\left(\bar{x}_{0}\right)^{2}-2 p \bar{x}_{0} \mu\left(s_{0}\right) .
\end{aligned}
$$

Hence,

$$
\begin{aligned}
\mathrm{E}\left\{J_{*}\left(\mathrm{f}_{X_{0}-\bar{x}_{0} \mid S_{0}}\right)\right\}= & (\alpha-p) \mathrm{E}\left\{\operatorname{var}_{S_{0}}\left\{X_{0}\right\}\right\}+p \mathrm{E}\left\{X_{0}^{2}\right\} \\
& +p\left(\bar{x}_{0}\right)^{2}-2 p \bar{x}_{0} \mathrm{E}\left\{X_{0}\right\}, \\
= & (\alpha-p) \mathrm{E}\left\{\operatorname{var}_{S_{0}}\left\{X_{0}\right\}\right\}+p \operatorname{var}\left\{X_{0}\right\},
\end{aligned}
$$

also using (11), where $p$ is given by (6) and $\alpha$ is the as-yet-undetermined constant of Lemma 2 . Similarly,

$$
J_{*}\left(\mathrm{f}_{X_{0}}\right)=\alpha \operatorname{var}\left\{X_{0}\right\}+p\left(\bar{x}_{0}\right)^{2} .
$$

Substituting this and (12) into Theorem 1, 
$\alpha \operatorname{var}\left\{X_{0}\right\}+p\left(\bar{x}_{0}\right)^{2}=a^{2} \operatorname{var}\left\{X_{0}\right\}+\frac{a^{2}(p+1) r}{(p+1) b^{2}+r}\left(\bar{x}_{0}\right)^{2} \begin{aligned} & \text { the optimal coder at time } t \text { simply partitions the } \\ & \text { support interval of } \mathrm{f}_{X_{t} \mid \tilde{s}_{t-1}} \text { into }|\mathcal{S}| \text { equal sub- }\end{aligned}$ $+a^{2} \inf _{\gamma_{0}}\left\{(\alpha-p) \mathrm{E}\left\{\operatorname{var}_{S_{0}}\left\{X_{0}\right\}\right\}+p \operatorname{var}\left\{X_{0}\right\}\right\}$.

By (6), this simplifies into

$\left[\alpha-a^{2}(1+p)\right] \operatorname{var}\left\{X_{0}\right\}=a^{2} \inf _{\gamma_{0}}(\alpha-p) \mathrm{E}\left\{\operatorname{var}_{S_{0}}\left\{X_{0}\right\}\right\}$.

There are two possibilities at this point, $\alpha \leq p$ or $\alpha>p$. Suppose first that $\alpha \leq p$. Then

$\left[\alpha-a^{2}(1+p)\right] \operatorname{var}\left\{X_{0}\right\}=a^{2}(\alpha-p) \sup _{\gamma_{0}} \mathrm{E}\left\{\operatorname{var}_{S_{0}}\left\{X_{0}\right\}\right\}$.

Recall that a standard property of means is that $\forall \psi \in \mathbb{R}, \mathrm{E}_{s_{0}}\left\{\left|X_{0}-\psi\right|^{2} \geq \mathrm{E}_{s_{0}}\left\{\left|X_{0}-\mu\left(s_{0}\right)\right|^{2}=\right.\right.$ $\operatorname{var}_{s_{0}}\left\{X_{0}\right\}$. Setting $\psi=\bar{x}_{0}$, it follows that for any coding function $\gamma_{0}$,

$$
\begin{aligned}
\operatorname{var}\left\{X_{0}\right\} & =\mathrm{EE}_{s_{0}}\left\{\left|X_{0}-\bar{x}_{0}\right|^{2}\right\}, \\
& \geq \mathrm{EE}_{s_{0}}\left\{\left|X_{0}-\mu\left(s_{0}\right)\right|^{2}\right\}=\mathrm{E}\left\{\operatorname{var}_{S_{0}}\left\{X_{0}\right\}\right\} .
\end{aligned}
$$

Furthermore, the left-hand side can be approached arbitrarily closely by a parametrised coder $\gamma_{0}^{n}$ such that $s_{0}=0$ iff $x_{0} \in[-n, n]$. As $n \rightarrow$ $\infty \mathrm{P}\left\{S_{0}=0\right\} \rightarrow 1, \mathrm{E}_{S_{0}=0}\left\{X_{0}\right\} \rightarrow \bar{x}_{0}$, $\mathrm{E}_{S_{0}=0}\left\{X_{0}^{2}\right\} \rightarrow \mathrm{E}\left\{X_{0}^{2}\right\}$, and consequently by (11) $\mathrm{E}\left\{\operatorname{var}_{S_{0}}\left\{X_{0}\right\}\right\} \rightarrow \operatorname{var}\left\{X_{0}\right\}$. Thus $\sup _{\gamma_{0}} \mathrm{E}\left\{\operatorname{var}_{S_{0}}\left\{X_{0}\right\}\right\}$ $=\operatorname{var}\left\{X_{0}\right\}$, which upon substitution into (14) yields

$$
\alpha-a^{2}(1+p)=a^{2}(\alpha-p) \Leftrightarrow \alpha=a^{2} /\left(1-a^{2}\right) .
$$

If $|a|>1$ this is clearly impossible, since $\alpha$ cannot be negative. On the other hand if $|a| \leq 1$ it can be shown from (6) that this contradicts the initial hypothesis $\alpha \leq p$.

Hence, $\alpha>p$ and (13) becomes

$\left[\alpha-a^{2}(1+p)\right] \operatorname{var}\left\{X_{0}\right\}=a^{2}(\alpha-p) \inf _{\gamma_{0}} \mathrm{E}\left\{\operatorname{var}_{S_{0}}\left\{X_{0}\right\}\right\}$.

As $\mathrm{E}\left\{\operatorname{var}_{S_{0}}\left\{X_{0}\right\}\right\}=\mathrm{E}\left\{\left|X_{0}-\mu\left(S_{0}\right)\right|^{2}\right\}$ is the mean square error in quantising a uniform distribution using $|\mathcal{S}|$ levels, it is straight-forward to show that the optimal quantiser is also uniform (see e.g. (Gersho and Gray, 1993)), yielding

$\mathrm{E}\left\{\operatorname{var}_{S_{0}}\left\{X_{0}\right\}\right\}=\mathrm{E}\left\{\left|X_{0}-\mu\left(S_{0}\right)\right|^{2}\right\}=\operatorname{var}\left\{X_{0}\right\} /|\mathcal{S}|^{2}$.

Substituting this into (15), it then follows that

$$
\alpha=a^{2}(1+p)+a^{2} \frac{a^{2}(1+p)-p}{|\mathcal{S}|^{2}-a^{2}} .
$$

The theorem then follows from (12).

Assuming a uniformly distributed initial condition, this formula gives the smallest possible cost which can be achieved with a given number of coding symbols. By inverting it, the minimum number of levels needed to obtain a given cost can also be found. Though not shown explicitly, it should be plain from (15) - (16) and (9) that intervals, as might be expected from the uniform distribution of the initial state.

Observe that the minimum cost $\rightarrow \infty$ as $|\mathcal{S}|-$ $|a| \rightarrow 0$, agreeing with results from (Nair and Evans, 2000; Nair and Evans, 2003). As a further check, note that since the quantisation errors become negligible as the channel alphabet size $|\mathcal{S}|$ grows, the channel-constrained optimal cost should approach the unconstrained mean classical optimal LQR cost. However, a point of difference with the usual LQR formulation is that the initial input $u_{0}=\delta_{0}$ is a known constant independent of the unknown initial state $x_{0}$, because the channel delay remains fixed at 1 . At any time $t \geq 1$ though, the controller receives a codeword $s_{t-1} \in$ $\mathcal{S}$ which encodes $x_{t-1}$ with arbitrarily high resolution, and consequently can calculate the value of $x_{t}=a x_{t-1}+b \delta_{t-1}\left(\tilde{s}_{t-2}\right)$ with high precision. Thus the classical LQR control law can only be applied from time $t \geq 1$, with associated deterministic cost $p x_{1}^{2}=p\left(a x_{0}+b \delta_{0}\right)^{2}$. The overall optimal cost for the unconstrained system is then obtained by adding the incremental cost $\left(a x_{0}+b \delta_{0}\right)^{2}+r \delta_{0}^{2}$ at $t=0$, averaging over the initial state and then minimising over $\delta_{0}$,

$$
\begin{aligned}
& J_{*}^{\text {ideal }}\left(\mathrm{f}_{X_{0}}\right) \\
& =\min _{\delta_{0}} \mathrm{E}\left\{p\left(a X_{0}+b \delta_{0}\right)^{2}+\left(a X_{0}+b \delta_{0}\right)^{2}+r \delta_{0}^{2}\right\} \\
& =a^{2}(p+1) \operatorname{var}\left\{X_{0}\right\} \\
& \quad+\min _{\delta_{0}}\left\{(p+1)\left(a \bar{x}_{0}+b \delta_{0}\right)^{2}+r \delta_{0}^{2}\right\}, \\
& =a^{2}(p+1) \operatorname{var}\left\{X_{0}\right\}+p\left(\bar{x}_{0}\right)^{2} .
\end{aligned}
$$

Comparison with Theorem 3 reveals that this is precisely the limiting optimal cost $J_{*}\left(f_{X_{0}}\right)$ as $|\mathcal{S}| \rightarrow \infty$. Furthermore, the optimal channelconstrained cost can then be written

$$
J_{*}\left(\mathrm{f}_{X_{0}}\right)=J_{*}^{\text {ideal }}\left(\mathrm{f}_{X_{0}}\right)+\frac{a^{2}(1+p)-p}{(|\mathcal{S}| / a)^{2}-1} \operatorname{var}\left\{X_{0}\right\},
$$

so the second term is the average loss of optimal performance due to a finite channel alphabet.

It is unlikely that the infimum cost expressions derived above also apply to general non-uniform pdf's. Nonetheless, the techniques used in this paper offer a way to obtain formulae for those classes of density functions that retain their form after conditioning on an interval.

For instance, consider the class $\mathcal{P}_{M}$ of densities that are piecewise constant on at most $M$ real intervals. Each pdf is completely specified by $2 M-2$ parameters, and it is a reasonable conjecture that on this class of densities $J_{*}\left(\mathrm{f}_{X}\right)$ is a quadratic function of these parameters. Thus, determining $J_{*}\left(\mathrm{f}_{X}\right)$ reduces to determining the constant co- 
efficients of this quadratic form. Then note that any such density remains an element of $\mathcal{P}_{M}$ when conditioned on an interval, since it can still take at most $M$ different values. The recursive equation of Theorem 1 and the scaling and shifting properties of $J_{*}$ can then be used to obtain exact formulae for each of these coefficients. If these formulae happen to retain a well-defined structure as $M \rightarrow \infty$, then this could also be a way to derive the infimum cost of arbitrary pdf's, by approximating them with sufficiently fine piecewise constant densities.

\section{CONCLUSION}

This paper studied the optimal data-rate-limited control of noiseless linear systems when the coder and controller are permitted to be time-varying with memory. The performance cost considered was the average quadratic regulation criterion. Several fundamental properties of the infimum cost functional were obtained under a mild moment condition and, using them, precise formulae for the optimal cost and policy were derived for the case of a uniformly distributed initial state. These expressions agreed with the classical optimal LQR results in the high data rate limit and with known minimum rate results in the low rate regime. To the best of the authors' knowledge they are the first exact characterisation of optimal data-rate-limited performance in any formulation. The derivation of analagous formulae for nonuniform distributions and vector-valued states remains an open problem.

\section{REFERENCES}

Baillieul, J. (2001). Feedback designs in information-based control. In: Stochastic Theory and Control Proceedings of a Workshop held in Lawrence, Kansas (B. PasikDuncan, Ed.). Springer. pp. 35-57.

Brockett, R. W. and D. Liberzon (2000). Quantized feedback stabilization of linear systems. IEEE Trans. Autom. Contr. 45(7), 1279-89.

Delchamps, D. F. (1990). Stabilizing a linear system with quantized state feedback. IEEE Trans. Autom. Contr. 35, 916-24.

Fagnani, F. and S. Zampieri (2003). Stability analysis and synthesis for scalar linear systems with a quantized feedback. IEEE Trans. Autom. Contr. 48(9), 1569-84.

Fagnani, F. and S. Zampieri (2004a). Quantized stabilization of linear systems: complexity versus performance. IEEE Trans. Autom. Contr. 49(9), 1534-48.

Fagnani, F. and S. Zampieri (2004b). Tree structured vector quantization and quantized control. Preprint.
Gersho, A. and R. M. Gray (1993). Vector Quantization and Signal Compression. Kluwer.

Ishii, H. and B. A. Francis (2003). Quadratic stabilization of sampled-data systems with quantization. Automatica 39(10), 1793-1800.

Li, K. and J. Baillieul (2004). Robust quantization for digital finite communication bandwidth (dfcb) control. IEEE Trans. Autom. Contr. 49(9), 1573-97.

Liberzon, D. (2003). On stabilization of linear systems with limited information. IEEE Trans. Autom. Contr. 48(2), 304-7.

Nair, G. N. and R. J. Evans (2000). Stabilization with data-rate-limited feedback: tightest attainable bounds. Sys. Contr. Lett. 41(1), 4956.

Nair, G. N. and R. J. Evans (2003). Exponential stabilisability of finite-dimensional linear systems with limited data rates. Automatica 39, 585-93.

Nair, G. N. and R. J. Evans (2004). Stabilizability of stochastic linear systems with finite feedback data rates. SIAM Jour. Contr. Optim. 43(2), 413-36. Short version published in Proc. 41st IEEE Conf. Dec. Contr., 2002.

Petersen, I. R. and A. V. Savkin (2001). Multirate stabilization of multivariable discretetime linear systems via a limited capacity communication channel. In: Proc. 40th IEEE Conf. Dec. Contr.. pp. 304-9.

Special Issue on Networked Control Systems (2004). IEEE Trans. Autom. Contr.

Tatikonda, S., A. Sahai and S. Mitter (2004). Stochastic linear control over a communication channel. IEEE Trans. Autom. Contr. 49(9), 1549-61.

Tatikonda, S. and S. Mitter (2000). Control under communication constraints. In: Proc. 38th Ann. Allerton Conf. Comm. Contr. Comp.. pp. 182-90.

Wong, W. S. and R. W. Brockett (1999). Systems with finite communication bandwidth constraints II: stabilization with limited information feedback. IEEE Trans. Autom. Contr. 44, 1049-53.

Yuksel, S., O. C. Imer and T. Basar (2004). Constrained state estimation and control over communication networks. In: Proc. 38th Ann. Conf. Inf. Sci. Syst. 